\title{
On convergence properties of variational constitutive updates for elasto-visco-plasticity
}

\section{Laurence Brassart ${ }^{1}$ and Laurent Stainier ${ }^{* 2}$}

${ }^{1}$ Université catholique de Louvain, iMMC

4 Av. Georges Lemaître, B-1348 Louvain-la-Neuve, Belgium

2 Ecole Centrale de Nantes, GeM

1 Rue de la Noë, BP 92101, F-44321 Nantes, France

Key words Variational updates, viscoplasticity, convergence.

MSC (2000) 74D10

This paper deals with $J_{2}$ elasto-visco-plasticity and analyzes in details a variational formulation of associated constitutive updates. The variational formulation is briefly presented and compared with the traditional radial return algorithm. Differences are highlighted in the case of combined hardening and rate-dependency models. In that case, the variational formulation introduces an algorithmic parameter, which effect is analyzed on precision and convergence behavior. A practical rule is proposed for choosing an optimal value for this parameter.

\section{Introduction}

With the generalization of numerical simulations involving more and more complex material behavior, integration of constitutive models has become an important part of many academic and industrial softwares dedicated to solving problems in structural or solid mechanics. This paper focuses on constitutive updates for materials whose local response can be described by classical $J_{2}$ elasto-visco-plastic theory with isotropic hardening. Thermal effects are not considered. The constitutive equations are written within the framework of Generalized Standard Media (GSM) [1,2]), according to which state laws and complementary laws for the evolution of the internal variables respectively derive from a free energy and a dissipation function.

Time integration of the constitutive equations along a given path of applied deformation is performed according to an incremental variational principle. Numerous authors contributed to the development of variational principles equivalent to the incremental formulation of elastovisco-plasticity (e.g.: Mialon [3]; Comi et al. [4]; Martin et al. [5]; Carini [6]; Miehe [7]). In particular, Ortiz and Stainier [8] proposed a unified incremental variational formulation in the general context of elasto-(visco)plasticity at finite strains. On a time interval, updates of internal variables are obtained from the minimization of a suitably chosen functional, involving the free energy and the dissipation function. The minimized functional acts in turn as a unique potential for the stress. 
The particular mathematical structure of these incremental updates, inherited from its variational nature, has been exploited in various directions such as mesh adaption $[9,10]$ or numerical and semi-analytical homogenization [7,11-13]. Variational constitutive updates are not limited to simple $J_{2}$ elasto-visco-plasticity and extensions have been proposed to more complex plasticity models $[14,15]$, non-linear viscoelasticity [16] or coupled thermo-mechanical boundary-value problems [17], to list only a few examples.

This paper details the application of variational constitutive updates to $J_{2}$ elasto-viscoplasticity with isotropic hardening under the assumption of linearized kinematics, and analyzes some of its numerical implications. Section 2 first presents constitutive equations in a thermodynamic framework adapted to the derivation of incremental variational updates (2.2). It is shown that the latter lead to an algorithmic approach similar in nature to the standard radial return of Wilkins [18]. Variational updates differ from the radial return in the case of coupled strain-hardening and rate-dependency models, for which the integration of dissipation terms over a finite time step by a generalized mid-point rule requires the introduction of an algorithmic parameter $\theta$ and introduces an additional term in the plastic yield equation. ${ }^{1}$ This additional term vanishes with small time (or load) increments, and the method thus remains consistent. For finite steps, the algorithmic parameter can be tuned to ensure that the yield condition is verified exactly at the end of the step, and an optimal value of the parameter is proposed (2.3). Section 3 illustrates the previous analysis for the case of combined power-law strain-rate dependence and linear or more general power-law strain hardening, for monotonous and cyclic uniaxial loading cases.

\section{Variational formulation of incremental viscoplastic updates}

\subsection{Thermodynamic framework}

In this paper, we consider material behavior which can be described by $J_{2}$ elasto-visco-plastic theory with isotropic hardening. Under the linearized kinematics hypothesis, the (symmetric) total strain tensor $\varepsilon$ is then classically decomposed into an elastic and a plastic part:

$$
\varepsilon=\varepsilon^{e}+\varepsilon^{p}
$$

The chosen set of state variables consists in the total strain $\varepsilon$, the plastic strain $\varepsilon^{p}$ and an additional scalar variable $p$ describing isotropic hardening and related to the accumulation of plastic deformation.

Contrarily to classical formulations (as described for instance in Lemaitre and Chaboche [20] or Maugin [21]), no yield function is explicitly introduced. Instead, kinematic restrictions related to the plastic flow are postulated a priori. Based on the expected plastic flow kinematics in von Mises plasticity, the rate of plastic strain is split into direction $N$ and amplitude $\dot{p}$ :

$$
\dot{\varepsilon}^{p}=\dot{p} \boldsymbol{N}
$$

1 A similar situation can be found in the case of variational updates for coupled thermo-mechanical viscoplasticity models, where specific precautions must be taken to preserve consistency with regard to the energy balance (i.e. heat) equation [19]. 
where $N$ is a kinematic variable ${ }^{2}$ satisfying the following constraints:

$$
\operatorname{tr}(\boldsymbol{N})=0 \quad \text { and } \quad \boldsymbol{N}: \boldsymbol{N}=\frac{3}{2}
$$

Constraints on $N$ ensure incompressibility of the plastic flow and uniqueness of decomposition (2). With such norm of $\boldsymbol{N}$, it is easy to check that: $\dot{p}=\left((2 / 3) \dot{\varepsilon}^{p}: \dot{\boldsymbol{\varepsilon}}^{p}\right)^{1 / 2}$, so that the scalar variable $p$ is the classical accumulated plastic strain. The kinematic variable $N$ will be specified later.

Supposing that the elastic response is independent of irreversible processes, the Helmholtz free energy (per unit volume) admits the following additive decomposition:

$$
\psi\left(\varepsilon, \varepsilon^{p}, p\right)=\psi^{e}\left(\varepsilon-\varepsilon^{p}\right)+\psi^{p}(p) .
$$

The elastic part $\psi^{e}$ represents the energy stored within the material and recoverable through elastic relaxation. A linear response in the elastic regime is obtained by taking $\psi^{e}$ quadratic in the elastic strain:

$$
\psi^{e}\left(\varepsilon-\varepsilon^{p}\right)=\frac{1}{2}\left(\varepsilon-\varepsilon^{p}\right): C^{e}:\left(\varepsilon-\varepsilon^{p}\right) .
$$

where $\boldsymbol{C}^{e}$ is a fourth-order elasticity tensor. The plastic part $\psi^{p}$ describes (isotropic) hardening by rate-independent and irreversible internal storage mechanisms and is written as:

$$
\psi^{p}(p)=\int_{0}^{p} R(q) d q
$$

where $R(p)$ represents a hardening stress (the function $R$ is supposed to be given) ${ }^{3}$. Kinematic hardening can be modeled by including a dependence of the plastic potential on the plastic strain $\varepsilon^{p}$. However, kinematic hardening is not considered here.

The state law for the stress is obtained from the free energy as:

$$
\boldsymbol{\sigma}=\frac{\partial \psi}{\partial \varepsilon}\left(\varepsilon, \varepsilon^{p}, p\right)=-\frac{\partial \psi}{\partial \varepsilon^{p}}\left(\varepsilon, \varepsilon^{p}, p\right)=\frac{\partial \psi^{e}}{\partial \varepsilon^{e}}\left(\varepsilon-\varepsilon^{p}\right) .
$$

Hence, the stress is the force associated with both the total strain and the plastic strain. Similarly, the hardening stress $R$ is the thermodynamic force associated with the internal variable $p$ :

$$
R=\frac{\partial \psi}{\partial p}\left(\varepsilon, \varepsilon^{p}, p\right)=\frac{\partial \psi^{p}}{\partial p}(p)
$$

State laws (7) and (8) must be supplemented by a kinetic relation providing the evolution of the internal variable $p$ (the evolution of $\varepsilon^{p}$ being given by the flow rule (2)). The complementary

2 The kinematic variable $\boldsymbol{N}$ represents the direction of plastic flow. It should not be confused with some kinematic variable for the description of kinematic hardening. Kinematic hardening is not considered in the present work.

3 A finer description of the partition of plastic work in stored and dissipated energies has been introduced in [22] (see also references therein). However, such considerations can be set aside within the isothermal framework considered here. 
law must ensure that the mechanical dissipation $\mathcal{D}$ is non-negative. Here, the dissipation expresses as (see for instance [20]):

$$
\mathcal{D}=\boldsymbol{\sigma}: \dot{\varepsilon}^{p}-R \dot{p} \geq 0 .
$$

The dissipation may conveniently be rewritten into a condensed form, accounting for the flow rule (2):

$$
\mathcal{D}=Y(\boldsymbol{N}) \dot{p}
$$

where the function $Y$ is defined, for a given state $\left\{\varepsilon, \varepsilon^{p}, p\right\}$, as:

$$
Y(\boldsymbol{N})=\boldsymbol{\sigma}: \boldsymbol{N}-R
$$

Expression (10) of the dissipation indicates that the new scalar quantity $Y$ is the force conjugate to $p$, when it is computed for the actual flow direction. Then, the evolution law for $p$ can be expressed as a kinetic relation between $Y$ and $\dot{p}$. Following the theory of Generalized Standard Materials (GSM), it is supposed to derive from a dissipation function $\phi(\dot{p})$ :

$$
Y=\frac{\partial \phi}{\partial \dot{p}}(\dot{p}), \quad \text { or, equivalently, } \quad \dot{p}=\frac{\partial \phi^{*}}{\partial Y}(Y),
$$

where $\phi^{*}$ is the convex dual of $\phi$ by Legendre transform:

$$
\phi^{*}(Y)=\sup _{\dot{p}}\{\dot{p} Y-\phi(\dot{p})\} .
$$

By choosing $\phi(\dot{p})$ non-negative, convex and such that $\phi(0)=0$, the mechanical dissipation (10) is necessarily non-negative. By the properties of Legendre transform, the following result holds:

$$
\phi(\dot{p})+\phi^{*}(Y)=Y \dot{p}=\mathcal{D} .
$$

Note that the dual functions $\phi$ and $\phi^{*}$ may also depend on other state variables, which act as parameters in the Legendre transformation (13). For example, in the following, we will consider a dissipation function of Perzyna-type [23]:

$$
\phi(\dot{p} ; p)= \begin{cases}\sigma_{Y} \dot{p}+\frac{\dot{p}_{0} D(p)}{n+1}\left(\frac{\dot{p}}{\dot{p}_{0}}\right)^{n+1} & \text { if } \dot{p} \geq 0 \\ +\infty & \text { otherwise }\end{cases}
$$

where the drag stress $D(p)$ induces isotropic hardening.

The kinematic variable $N$ was not specified up to now. Actually, it can be shown that $N$ is found by maximizing the dissipation at fixed $p$ and $\dot{p}$. This follows from a continuous variational principle introduced by Ortiz and Stainier [8], which is not presented here. The kinematic variable will be specified within the discretized formulation presenter hereafter. 


\subsection{Incremental variational formulation}

We now consider the problem of integrating the constitutive relations over a time increment $\left[t_{k}, t_{k+1}\right]$. The state at $t_{k}$ is supposed to be given: $\left\{\varepsilon_{k}, \varepsilon_{k}^{p}, p_{k}\right\}$, as well as the total deformation at $t_{k+1}: \varepsilon_{k+1}$. We aim to compute the stress $\boldsymbol{\sigma}_{k+1}$, the plastic strain $\varepsilon_{k+1}^{p}$ and the accumulated plastic strain $p_{k+1}$ at $t_{k+1}$. We first assume that the rate of accumulated plastic strain $\dot{p}$ is constant over the time step and given by the difference quotient $\Delta p / \Delta t$, with $\Delta()=()_{k+1}-$ ()$_{k}$. Similarly, the plastic flow rule (2) is discretized as:

$$
\Delta \varepsilon^{p}=\Delta p N
$$

where $N$ is an (a priori unknown) constant plastic flow direction for the time step. Ortiz and Stainier [8] proposed the following incremental variational principle:

$$
W_{\Delta}\left(\varepsilon_{k+1}\right)=\inf _{\Delta p, \boldsymbol{N}} J_{\Delta}\left(\varepsilon_{k+1}, \Delta p, \boldsymbol{N}\right),
$$

where the minimization w.r.t. $\boldsymbol{N}$ is performed under constraints (3) and

$$
J_{\Delta}\left(\varepsilon_{k+1}, \Delta p, \boldsymbol{N}\right)=\psi\left(\varepsilon_{k+1}, \varepsilon_{k+1}^{p}, p_{k+1}\right)-\psi_{k}+\Delta t \phi\left(\frac{\Delta p}{\Delta t} ; p_{k+\theta}\right),
$$

where $\varepsilon_{k+1}^{p}$ is obtained from the discretized flow rule (16) and $\psi_{k}$ is the free energy computed for the (given) state variables at $t_{k}$. The dependence of $\phi$ in $p$ is handled by evaluating the dissipation function at an intermediary value during the time interval:

$$
p_{k+\theta}=(1-\theta) p_{k}+\theta p_{k+1}, \quad \theta \in[0,1] .
$$

Then, considering the stationarity conditions w.r.t. $\Delta p$ and $N$ in (17), the stress tensor at $t_{k+1}$ is given by:

$$
\boldsymbol{\sigma}_{k+1}=\frac{d W_{\Delta}}{d \varepsilon_{k+1}}\left(\varepsilon_{k+1}\right)=\frac{\partial J_{\Delta}}{\partial \varepsilon_{k+1}}\left(\varepsilon_{k+1}, \Delta p, \boldsymbol{N}\right),
$$

where $\Delta p$ and $N$ are the solutions of the minimization problem (17). Thus, the function $W_{\Delta}$ plays the role of an incremental potential for the stress. In the following we show that optimality conditions w.r.t. $\Delta p$ and $\boldsymbol{N}$ yield the classical incremental relations of $J_{2}$ plasticity. In particular, the well-known radial return scheme with its predictor and corrector steps ( [18], [24] or [25]) can be retrieved.

Taking into account the discretized flow rule (16), the stationarity condition of $J_{\Delta}$ w.r.t. $\Delta p$ gives the discretized kinetic relation (12):

$$
Y_{k+1}\left(\boldsymbol{N}, p_{k+1}\right)=\frac{\partial \phi}{\partial \dot{p}}\left(\frac{\Delta p}{\Delta t} ; p_{k+\theta}\right)+\theta \Delta t \frac{\partial \phi}{\partial p}\left(\frac{\Delta p}{\Delta t} ; p_{k+\theta}\right),
$$

where $\partial \phi / \partial \dot{p}$ and $\partial \phi / \partial p$ have to be interpreted as functions obtained by derivation of the continuous function $\phi$ with respect to its first and second arguments, and evaluated with the finite difference approximation of $\dot{p}$. The function $Y_{k+1}$ is defined similarly as in the continuous case (11):

$$
Y_{k+1}\left(\boldsymbol{N}, p_{k+1}\right) \equiv \boldsymbol{\sigma}_{k+1}: \boldsymbol{N}-R\left(p_{k+1}\right) .
$$


Note that $\boldsymbol{\sigma}_{k+1}$ now depends on $\boldsymbol{N}$, since:

$$
\boldsymbol{\sigma}_{k+1}=\frac{\partial \psi^{e}}{\partial \varepsilon^{e}}\left(\varepsilon_{k+1}^{e}\right), \quad \text { with } \quad \varepsilon_{k+1}^{e}=\varepsilon_{k+1}-\varepsilon_{k}^{p}-\Delta p \boldsymbol{N}
$$

Therefore, it is conveniently rewritten as:

$$
\boldsymbol{\sigma}_{k+1}=\boldsymbol{C}^{e}:\left(\varepsilon_{k+1}^{\mathrm{tr}}-\Delta p \boldsymbol{N}\right)=\boldsymbol{\sigma}_{k+1}^{\mathrm{tr}}-\Delta p\left(\boldsymbol{C}^{e}: \boldsymbol{N}\right),
$$

where the trial (or predictor) elastic strain $\varepsilon_{k+1}^{\mathrm{tr}}$ and the corresponding trial stress $\boldsymbol{\sigma}_{k+1}^{\mathrm{tr}}$ were introduced:

$$
\begin{aligned}
\varepsilon_{k+1}^{\mathrm{tr}} & \equiv \varepsilon_{k+1}-\varepsilon_{k}^{p}, \\
\sigma_{k+1}^{\mathrm{tr}} & \equiv C^{e}: \varepsilon_{k+1}^{\mathrm{tr}} .
\end{aligned}
$$

The minimization of $J_{\Delta}$ w.r.t. $N$ under constraints (3) is performed using Lagrange multipliers and yields

$$
N=\frac{3}{2} \frac{s_{k+1}^{\mathrm{tr}}}{\sigma_{\mathrm{eq}, n+1}^{\mathrm{tr}}}=\frac{\boldsymbol{e}^{\mathrm{tr}}}{\varepsilon_{\mathrm{eq}}^{\mathrm{tr}}}
$$

Expression (27) of the kinematic variable $\boldsymbol{N}$ is obtained assuming isotropic elasticity, in which case the elastic stiffness tensor admits the following decomposition:

$$
\boldsymbol{C}^{e}=3 \kappa \boldsymbol{I}^{\mathrm{vol}}+2 \mu \boldsymbol{I}^{\mathrm{dev}},
$$

where $\kappa$ and $\mu$ are the elastic bulk and shear moduli, respectively. The spherical and deviatoric operators $\boldsymbol{I}^{\mathrm{vol}}$ and $\boldsymbol{I}^{\mathrm{dev}}$ are given by:

$$
\boldsymbol{I}^{\mathrm{vol}} \equiv \frac{1}{3} \mathbf{1} \otimes \mathbf{1}, \quad \boldsymbol{I}^{\mathrm{dev}} \equiv \boldsymbol{I}-\boldsymbol{I}^{\mathrm{vol}}
$$

where the symbols $\mathbf{1}$ and $\boldsymbol{I}$ stand for the second and symmetric fourth order identity tensors, respectively. The von Mises measures of stress and strain are respectively given by:

$$
\sigma_{\mathrm{eq}}=\left(\frac{3}{2} s: s\right)^{1 / 2} \quad \text { and } \quad \varepsilon_{\mathrm{eq}}=\left(\frac{2}{3} e: e\right)^{1 / 2}
$$

where $s$ and $e$ denote the deviatoric parts of $\sigma$ and $\varepsilon$ :

$$
s=I^{\mathrm{dev}}: \sigma, \quad e=\boldsymbol{I}^{\mathrm{dev}}: \varepsilon .
$$

Substituting (27) into (22), the stationnarity condition (21) for $\Delta p$ becomes:

$$
-3 \mu \varepsilon_{\mathrm{eq}, n+1}^{\mathrm{tr}}+3 \mu \Delta p+R\left(p_{k+1}\right)+\frac{\partial \phi}{\partial \dot{p}}\left(\frac{\Delta p}{\Delta t} ; p_{k+\theta}\right)+\underbrace{\theta \Delta t \frac{\partial \phi}{\partial p}\left(\frac{\Delta p}{\Delta t} ; p_{k+\theta}\right)}_{\mathcal{O}(\Delta t)}=0 .
$$

The problem of the non-smoothness of the dissipation function for $\Delta p=0$ can be circumvented by first evaluating the slope of the functional $J_{\Delta}$ for $\Delta p=0^{+}$. If it is negative, that is:

$$
-3 \mu \varepsilon_{\mathrm{eq}, n+1}^{\mathrm{tr}}+R\left(p_{k}\right)+\frac{\partial \phi}{\partial \dot{p}}\left(0^{+} ; p_{k}\right)<0
$$


then the optimal $\Delta p$ is positive, and satisfies condition (32). Otherwise, the optimal $\Delta p$ is zero, as negative values are prohibited (they would lead to infinite dissipation), and the increment is elastic. Therefore, the minimization problem associated with the incremental variational principle involves the evaluation of a yield criterion in terms of an elastic predictor, and a plastic correction step, exactly like in the classical radial return scheme. Differences arise from the presence of the integration parameter $\theta$ and the additional term in $\mathcal{O}(\Delta t)$, which becomes negligible for sufficiently small time increments and can be cancelled by a proper choice of $\theta$. Such numerical aspects are discussed in the next section.

\subsection{Numerical considerations}

The numerical scheme for the update of $\Delta p$ derived from the incremental variational principle can be compared to the classical radial return equations. For rate-independent plasticity $(D(p)=0$ in (15)), the dissipation function presents no parametric dependence in $p$. Hence condition (32) then simply becomes:

$$
-3 \mu \varepsilon_{\mathrm{eq}, n+1}^{\mathrm{tr}}+3 \mu \Delta p+R\left(p_{k+1}\right)+\underbrace{\frac{\partial \phi}{\partial \dot{p}}\left(\frac{\Delta p}{\Delta t}\right)}_{=\sigma_{Y} \text { if } \Delta p>0}=0,
$$

which coincides exactly with the classical radial return condition. Note that the above result assumes that hardening mechanisms contribute only to stored plastic energy, which may not correspond to what is experimentally observed. A more general treatment of rate-independent hardening, accounting for the partition between storage and dissipation mechanisms, is provided in [22]. Inclusion of dissipative rate-independent hardening terms leads to numerical effects very similar to those observed for rate-dependent (thus necessarily dissipative) hardening terms, and detailed in the remaining of this paper. For the sake of clarity, we will then limit ourselves to the latter case.

In the case of rate-dependent elasto-plasticity, the dissipation function may include a parametric dependence in $p$ (e.g. (15)). The update of accumulated plastic strain $\Delta p$ then obeys the discretized kinetic relation (21). Clearly, this discretized flow rule does not coincide with its continuous expression computed for the time $t_{k+1}$ :

$$
Y_{k+1}=\frac{\partial \phi}{\partial \dot{p}}\left(\frac{\Delta p}{\Delta t} ; p_{k+1}\right)
$$

which is fully-implicit, contrarily to expression (21) (at least, for arbitrary $\theta$ ).

Consider the specific case of a Perzyna-type dissipation function (15). According to equation (21), the force $Y_{k+1}$ is then given by:

$$
\begin{aligned}
Y_{k+1}=\sigma_{Y}+ & D\left(p_{k+\theta}\right)\left(\frac{\Delta p}{\dot{p}_{0} \Delta t}\right)^{n}+\theta \Delta t D^{\prime}\left(p_{k+\theta}\right) \frac{\dot{p}_{0}}{n+1}\left(\frac{\Delta p}{\dot{p}_{0} \Delta t}\right)^{n+1} \\
& =\sigma_{Y}+\left[D\left(p_{k+\theta}\right)+\frac{\theta \Delta p}{n+1} D^{\prime}\left(p_{k+\theta}\right)\right]\left(\frac{\Delta p}{\dot{p}_{0} \Delta t}\right)^{n} .
\end{aligned}
$$

On the other hand, fully-implicit discretization (35) of the continuous flow rule yields

$$
Y_{k+1}=\sigma_{Y}+D\left(p_{k+1}\right)\left(\frac{\Delta p}{\dot{p}_{0} \Delta t}\right)^{n} .
$$


To make expression (37) coincide with the fully implicit expression (38), the integration parameter $\theta$ should be chosen such that:

$$
D\left(p_{k+\theta}\right)+\frac{\theta \Delta p}{n+1} D^{\prime}\left(p_{k+\theta}\right)=D\left(p_{k+1}\right) .
$$

In the case where the drag stress is linear in $p: D(p)=\sigma_{Y}+h p$, the integration parameter corresponding to a fully implicit scheme is determined analytically from the previous equality, which is now rewritten as:

$$
h p_{k+\theta}+\frac{\theta \Delta p}{n+1} h=h p_{k+1} .
$$

It is readily found that $\theta$ must be chosen as:

$$
\theta=\frac{n+1}{n+2} \equiv \theta^{*}
$$

The optimal integration parameter is thus found to depend only on the rate-sensitivity exponent $n$, and not on any other material parameter. It is also independent from the current increment of the internal variable $\Delta p$. As the rate-sensitivity exponent ranges from 0 (rateindependent behavior) to 1 (linear rate-sensitivity), the integration parameter ranges from $\theta^{*}=1 / 2$ to $\theta^{*}=2 / 3$. For nonlinear hardening, equation (39) admits no analytical solution, and $\theta$ depends on the current value of $p$ and $\Delta p$. A detailed convergence study, analyzing the influence of the choice of $\theta$ on the integration error, is presented in next section.

\section{Convergence study}

We study the influence of the integration parameter $\theta$ on the convergence of the radial return scheme in the case of the Perzyna-type dissipation function (15) with power-law drag stress:

$$
D(p)=\sigma_{Y}+h p^{m} .
$$

We further assume that isotropic hardening comes exclusively from the dissipation function through the drag stress, so that $\psi^{p}(p)=R(p)=0$. Material parameters are the following: $E=100 \mathrm{GPa}, \nu=0.3, \sigma_{Y}=100 \mathrm{MPa}$ and $h=100 \mathrm{MPa}$.

We consider uniaxial tension/compression tests along direction 1 under applied strain rate $\left|\dot{\varepsilon}_{11}\right|=1 \mathrm{~s}^{-1}$. For a given number of loadsteps $N$, the relative error on the internal variable $p$ is defined as $\left|\left(p-p^{\text {ref }}\right)\right| / p^{\text {ref }}$, where $p^{\text {ref }}$ is the reference measure obtained with a very high number of time increments (here, $N=10000$ ).

Two sources of errors must be distinguished:

1. The discretization error, which may be reduced by increasing the number of time increments. As shown in the following, it as a major impact during the elastic-viscoplastic transitions.

2. An integration error related to the estimation of the dissipation function on a time interval. This error depends on the choice of $\theta$ in the generalized mid-point rule. This error is in $\mathcal{O}(\Delta t)$ (equation (32)) and is expected to be of little importance when $\Delta t$ is small. 
The first error is inherent to the incremental nature of numerical approaches, and it will be present in all formulations, including classical ones, based on satisfaction of an explicit yield condition at the end of the time step. The second error is proper to the variational approach. The so-called optimal integration parameter $\theta^{*}$ defined in (41) aims at canceling this second source of error.

\subsection{Linear hardening}

\subsubsection{Monotonic tension}

We consider first the case of linear hardening $(m=1)$, for several rate sensitivity exponents $n$. The stress-strain response for $n=0.5$ is presented in Figure (1(a)), and the relative error on $p$ at the end of the loading $\left(\varepsilon_{11}=0.05\right)$ as a function of the number of loadsteps $N$ for different values of $\theta$ is presented in Figure 1(b). For any $\theta$ chosen between 0 and 1 , the convergence is linear, as expected, except for the optimal integration parameter $\theta^{*}$ given by equation (41), for which the exact solution (at machine precision) is found as soon as $N$ becomes large enough to capture the elastic-viscoplastic transition. Similar convergence plots are obtained for other viscous exponents, but are not shown for conciseness.

The existence of an optimal integration parameter which depends on the viscous exponent is better demonstrated by plotting the relative error on $p$ at the end of the loading as a function of $\theta$ itself. Figure 2 presents the results for $n=0.5$, showing that the final relative error on the internal variable is minimized using $\theta=\theta^{*}$, no matter the number of loadsteps. The same conclusion holds for other rate-sensitivity exponents.

A better insight into the integration error may be given looking at the ratio $p / p^{\text {ref }}$. The numerical procedures overestimates (resp. underestimates) $p$ for $\theta<\theta^{*}$ (resp. $\theta>\theta^{*}$ ) (Figure 3), while the exact opposite holds regarding the stress (not shown). Indeed, the lefthand side of (40) can be rewritten as:

$$
h p_{k+\theta}+\frac{\theta \Delta p}{n+1} h=h\left(p_{k+1}+\left(\frac{\theta}{\theta^{*}}-1\right) \Delta p\right),
$$

from which it is clear that the drag stress contribution is overestimated when $\theta>\theta^{*}$.

\subsubsection{Tension/compression}

The stress response of the same material under a tension/compression test is presented in Figure 4 for a rate-sensitivity exponent $n=0.5$. We consider $N$ loadsteps on each quarter of cycle, and measure the error on $p$ at the end of the load cycle. The corresponding convergence graph (Figure 5) looks very different from the one obained in the monotonic case. Two distinct regions are observed:

- For $N \leq 50$, the error is essentially constant for $\theta=\theta^{*}$, while curves corresponding to $\theta>\theta^{*}$ (resp. $\theta<\theta^{*}$ ) show slightly decreasing (resp. increasing) error.

- For $N>50$, the error decreases sublinearly for all values of $\theta$, and the curves are hardly distinguishable. 


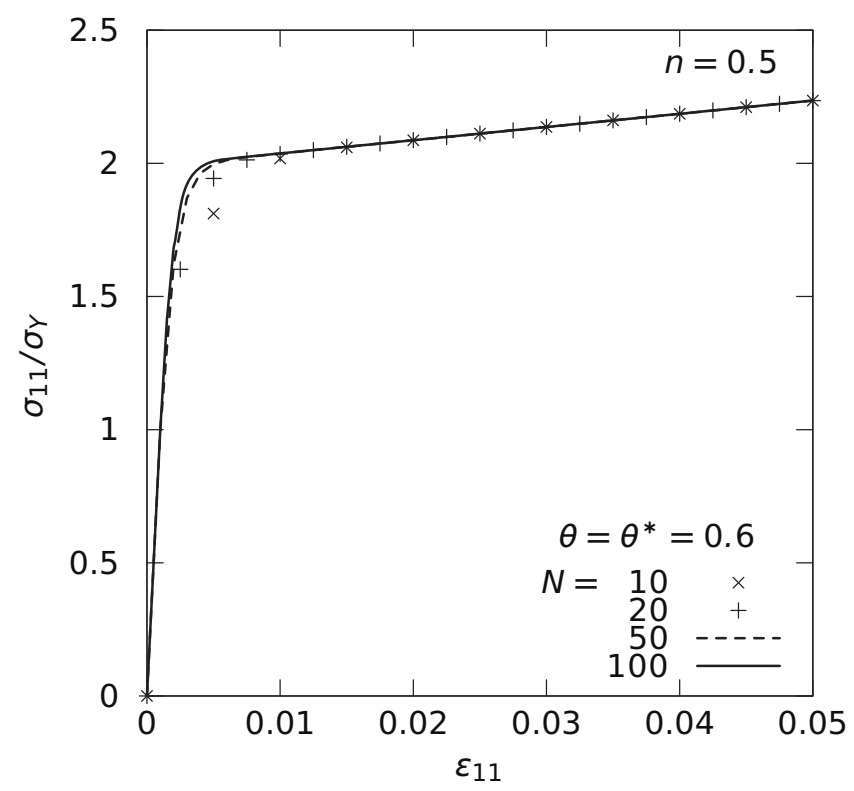

(a)

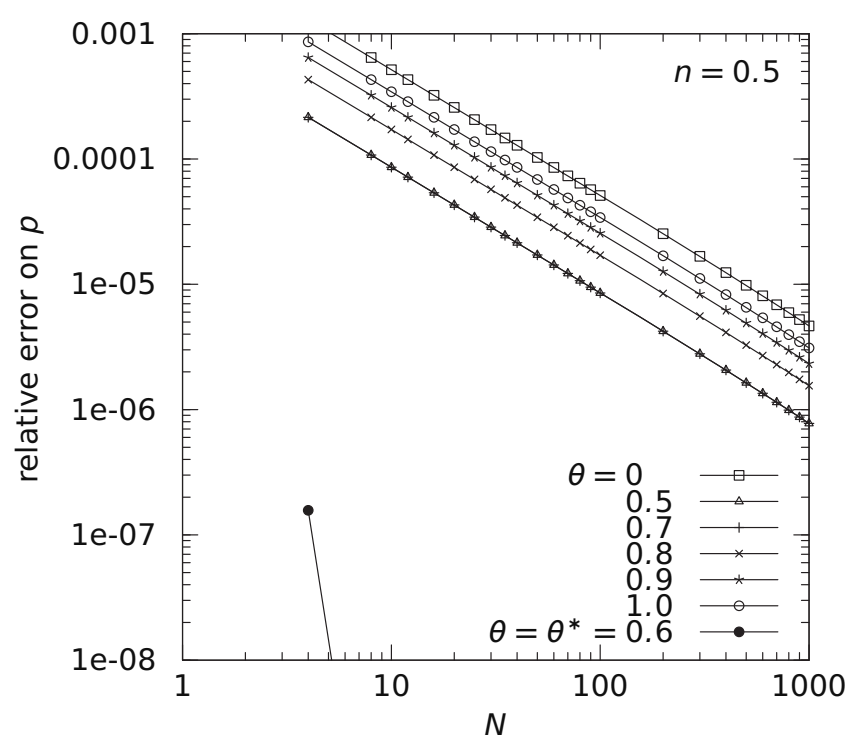

(b)

Fig. 1 (a) Normalized stress strain response of an elasto-viscoplastic material with linear drag stress $\left(D(p)=\sigma_{Y}+h p\right)$ under uniaxial tension $\left(\dot{\varepsilon}_{11}=1 \mathrm{~s}^{-1}\right)$. The discretization error is visible only during the elastic-viscoplastic transition. (b) Convergence plot for varying integration parameters $\theta$. The convergence is linear for $\theta \neq \theta^{*}$.

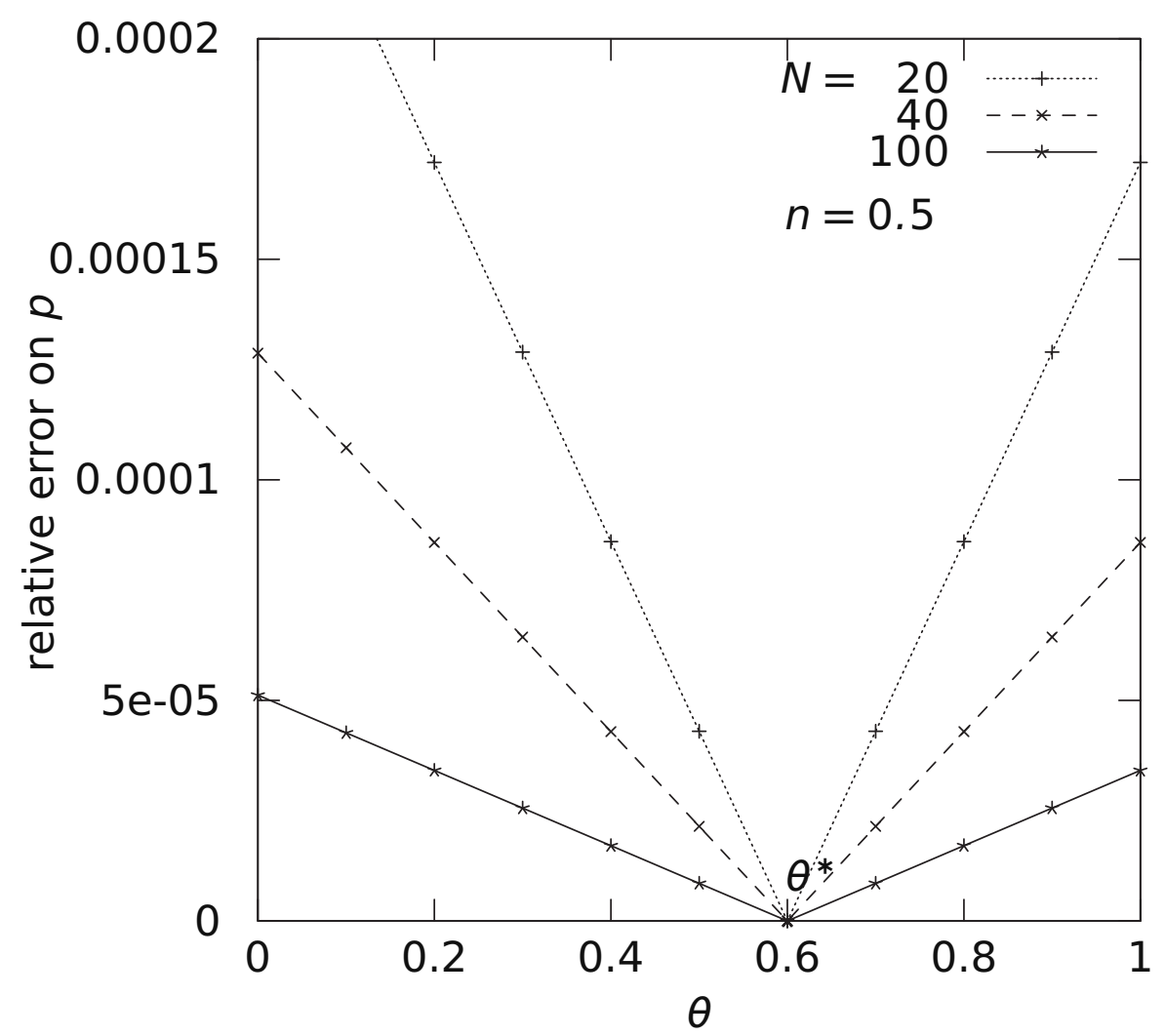

Fig. 2 Material with linear drag stress under uniaxial tension with rate-sensitivity exponent $n=0.5$. The optimal value of the integration parameter $\theta$ is given by: $(n+1) /(n+2)$. 


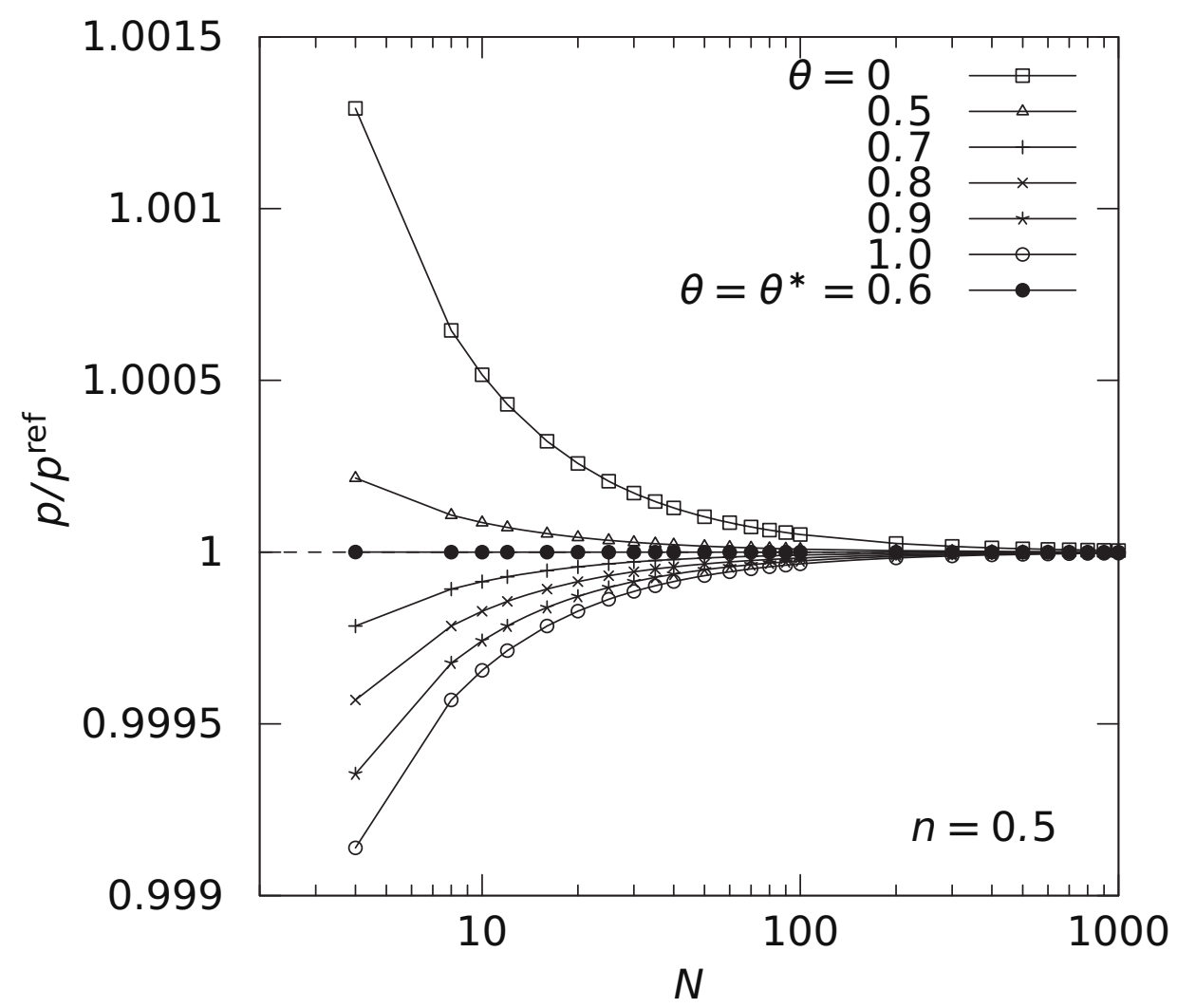

Fig. 3 Material with linear drag stress and rate-sensitivity exponent $n=0.5$ under uniaxial tension. Depending on the value of the integration parameter, the accumulated plastic strain is overestimated $\left(\theta<\theta^{*}\right)$ or underestimated $\left(\theta>\theta^{*}\right)$. The exact opposite observation holds regarding the axial stress (not shown).

These trends are related to the viscoplastic unloading taking place when the sign of the applied loading rate is reversed. During viscoplastic unloading, the accumulated plastic strain keeps increasing, and the slope of the curve is not the elastic one. The unloading remains viscoplastic until the stress reaches the yield surface, and becomes then purely elastic. The viscoplastic unloading step is captured by the incremental model only for very small time steps; otherwise, the sample unloads in a purely elastic way, thus introducing errors on the final value of $p$. Consequently, as long as the time steps number $N$ is not chosen large enough, the model misses the viscoplastic unload, and the relative error on $p$ stagnates.

Figure 6 shows the evolution of the ratio $p / p^{\text {ref }}$ at the end of the cycle with the number of load steps $N$. For small $N(N<50)$ the pattern is very similar to the one observed in Figure 3 , but shifted downward, as all curve underestimate the plastic strain level here. As in the uniaxial case, taking $\theta=0$ yields the higher estimate for $p$, so that the fully explicit estimate is the better one when considering the error on $p$. It can also be shown that the best estimate for the stress is obtained taking $\theta=1$. Therefore, no optimal value of $\theta$ can rigorously be defined. Note that for small $N$, the integration error is of the same order of magnitude than the discretization error, so that both can compensate in some way. For large $N$ (and small $\Delta t$ ), the discretization error is predominant, so that the particular choice of $\theta$ has a very limited impact on the final error. 


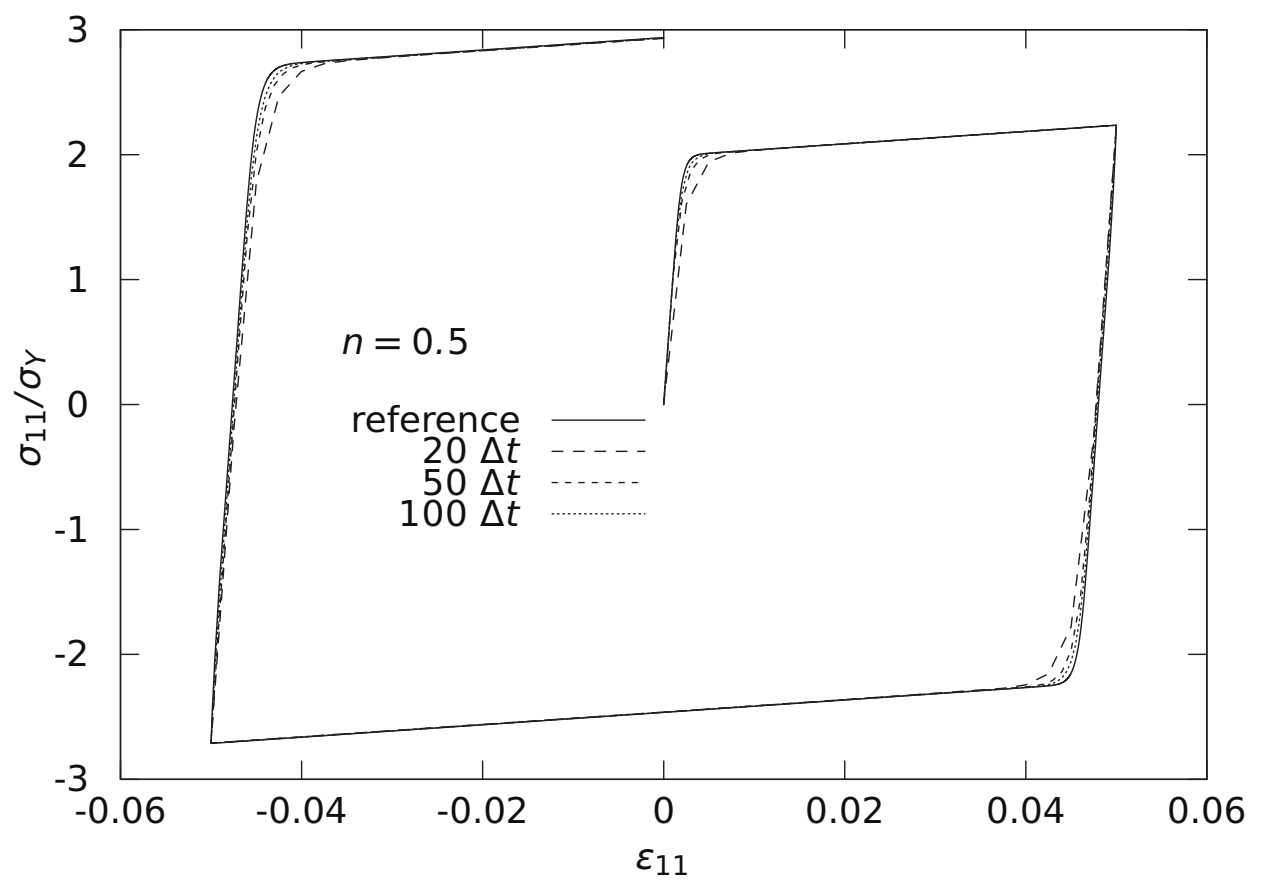

Fig. 4 Normalized stress strain response of an elasto-viscoplastic material with linear drag stress and rate-dependency exponent $n=0.5$ under uniaxial tension/compression $\left(\dot{\varepsilon}_{11}=1 \mathrm{~s}^{-1}\right)$.

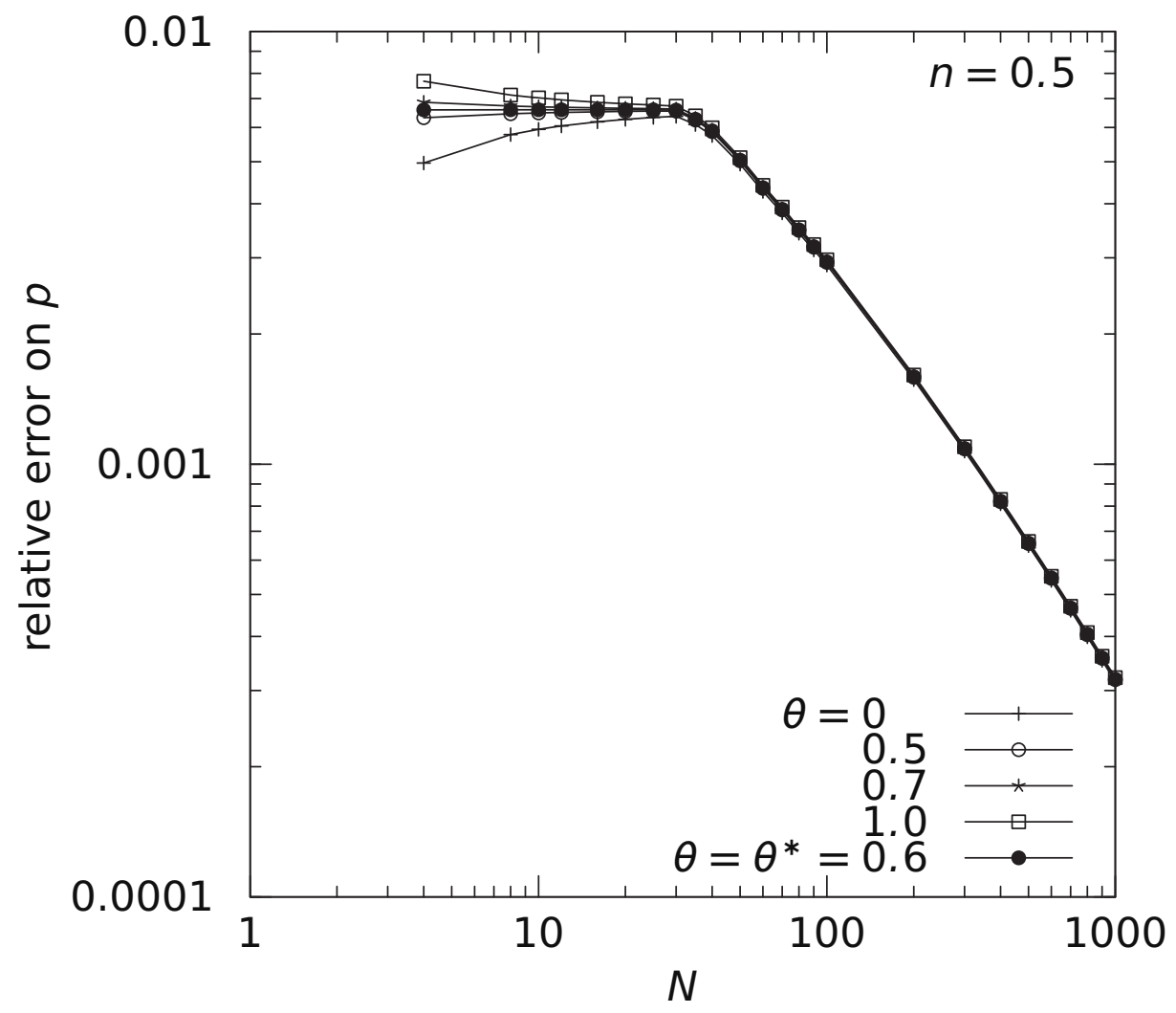

Fig. 5 Material with linear drag stress and rate-dependency exponent $n=0.5$ under uniaxial tension/compression. Convergence plots for varying integration parameters $\theta$. A minimal number of load steps is necessary in order to reach (sublinear) convergence. Contrarily to the uniaxial case, there is no optimal $\theta$ for which the final error is zero. 


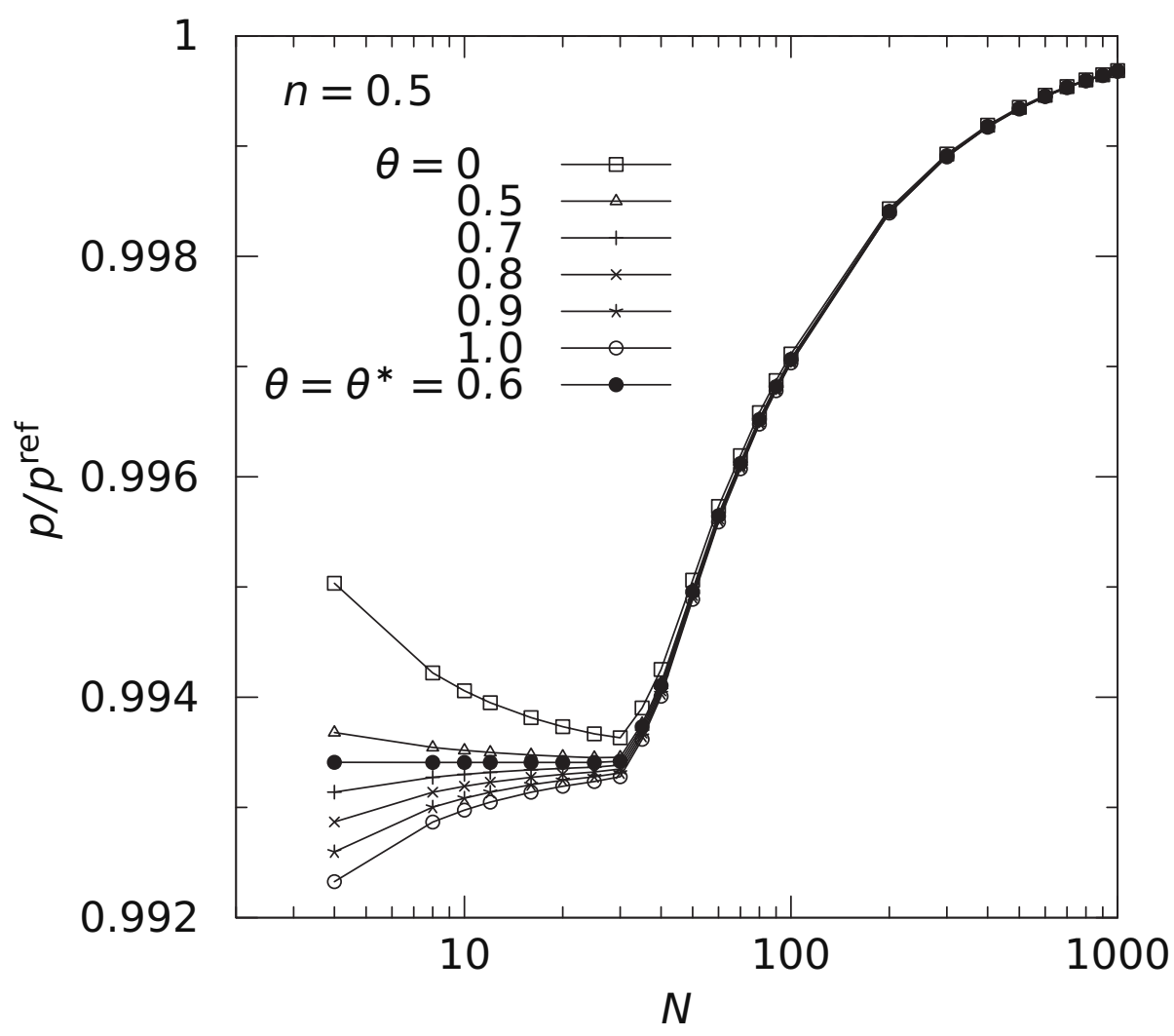

Fig. 6 Material with linear drag stress and rate-dependency exponent $n=0.5$ under uniaxial tension/compression. Taking $\theta=0$ yields the best estimate for $p$ when $N$ is small.

In order to study the error evolution during loading, we introduce an accumulated error measure:

$$
\text { cumulated relative error }=\sum_{N} \frac{\left|\Delta p-\Delta p^{\mathrm{ref}}\right|}{p^{\mathrm{ref}}}
$$

where $\Delta p^{\text {ref }}$ is the reference plastic increment computed from the reference simulation, and $p^{\text {ref }}$ is the reference accumulated plastic strain at the end of loading. The cumulated error is plotted in Figure 7 for $\theta=\theta^{*}$. As expected, error increases occur mostly during viscoplastic transition (during loadings and unloadings), while they are negligible during the purely elastic and purely plastic loadings. A similar behavior is expected under non-radial loading: major contributions to the integration error are expected around points where the loading mode is changing.

\subsection{Non-linear hardening}

We consider now the power-law drag stress (42) with exponent $m=0.5$. The integration parameter for a fully implicit scheme is found solving equation (39) numerically for the current value of $\Delta p$. The solution of the nonlinear equation is denoted by $\hat{\theta}$, while we keep the notation $\theta^{*}$ for the parameter (41) computed in the case of linear hardening. All other material properties are the same as in the previous examples, so as the applied strain rate.

All schemes obtained with different values of $\theta$ present a linear convergence w.r.t. $p$ (Figure $8(\mathrm{a})$ ). In particular, the convergence rate for $\theta=\hat{\theta}$ is also linear, but the error for large $\Delta t$ 


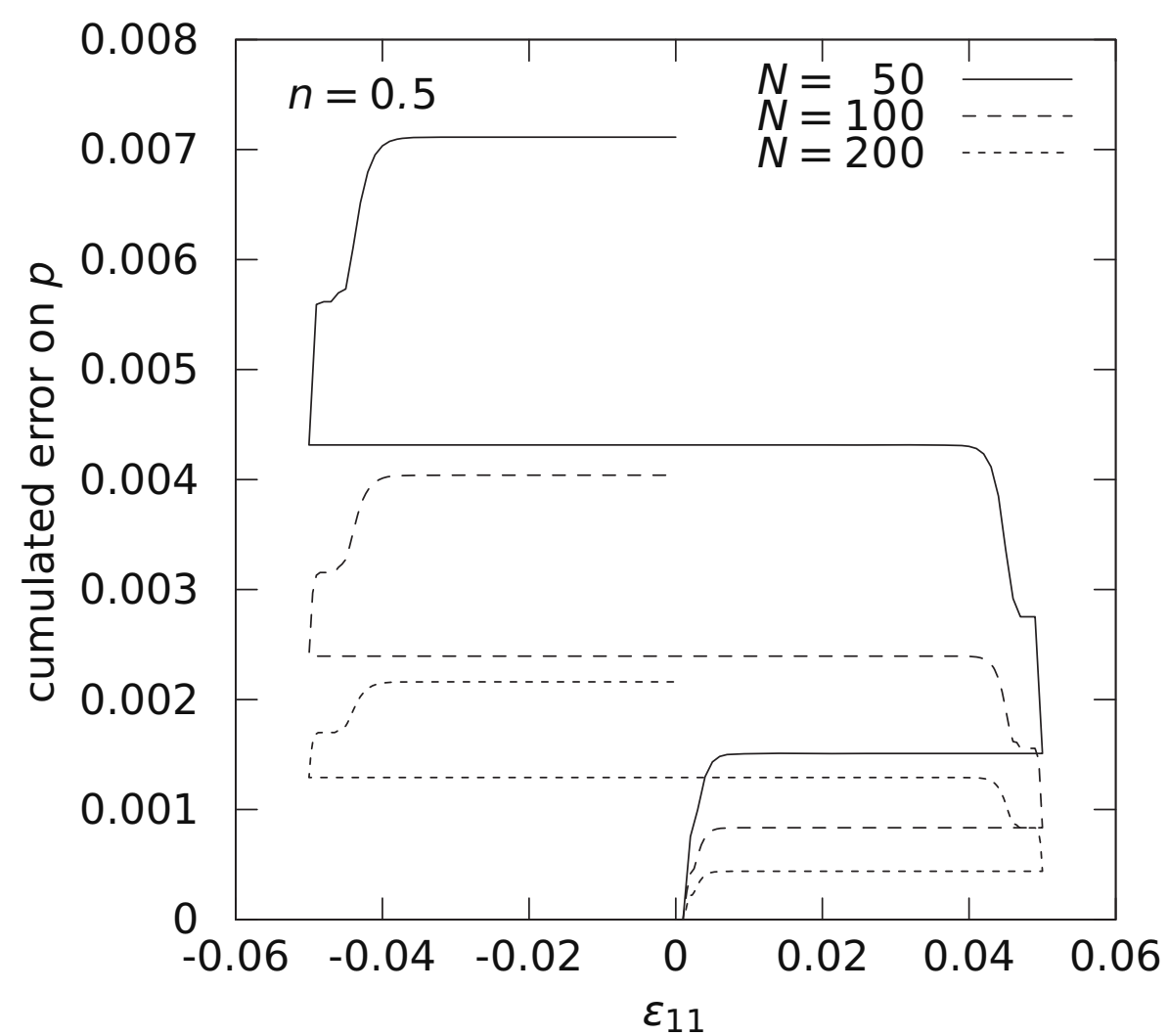

Fig. 7 Material with linear drag stress and rate-dependency exponent $n=0.5$ under uniaxial tension/compression. Evolution of the accumulated error during loading. Major error increases occur at elastic-viscoplastic transitions in both loading and unloading.

is lower than with other integration parameters (see also Figure 8(b)), except with $\theta=\theta^{*}$. Surprisingly, the convergence curve is non-monotonic for $\theta^{*}$, with a quadratic convergence rate at very small $N$. A close look to Figure (9(a)) shows that the error on $p$ when taking $\theta=\theta^{*}$ changes sign with $N$, which explains the non-monotonicity of the convergence plot in Figure 8(a). We conjecture that, for this particular value, the integration error compensates somewhat the discretization error, leading to better predictions than with $\hat{\theta}$ for small $N$. On the other hand, the fully-implicit solution with $\theta=\hat{\theta}$ overestimates $p^{\text {ref }}$ for any $N$.

The evolution of $\hat{\theta}$ during loading is presented in Figure 9 (b) for different $N$. The optimal value is very close to the expression obtained in the case of linear hardening, and the discrepancy between $\theta^{*}$ and $\hat{\theta}$ decreases with increasing numbers of loadsteps. The larger difference is observed during the elastic-plastic transition for all values of $N$.

\section{Conclusion}

When applied to $J_{2}$ plasticity with isotropic hardening, the incremental variational principle proposed by Ortiz and Stainier [8] yields a scalar radial return equation for the plastic strain update. Depending on the specific form of the dissipation function, the discretized constitutive equations may be completely equivalent to a classical, fully-implicit formulation, or may comprise an additional integration error. The integration error is function of the integration parameter $\theta$. For the dissipation function considered here (15), we suggest to use expression (41) for the integration parameter, which cancels the integration error in case of linear 
hardening. The same expression should be used also in the case of nonlinear hardening, as the theoretical value of $\theta$ obtained in this case (solution of equation (39)) does not yield significantly better predictions for a given loadstep size. For other models of rate-dependence

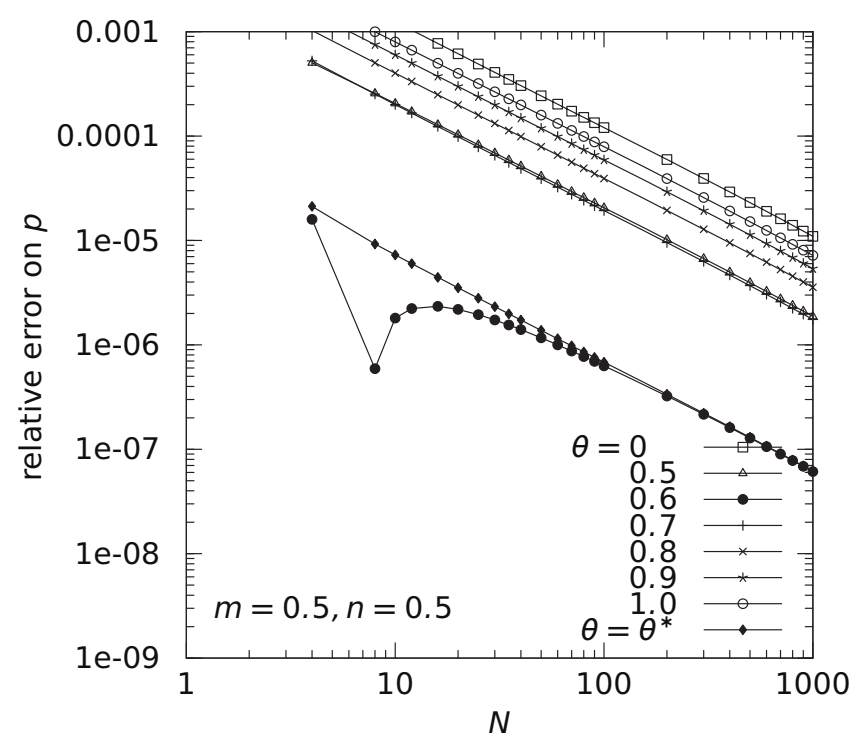

(a)

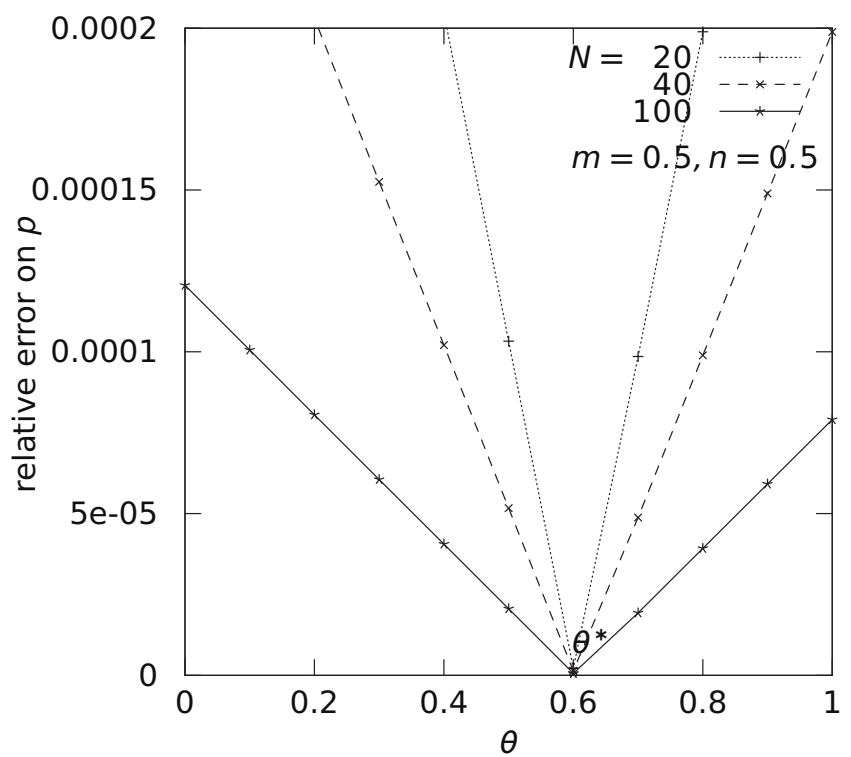

(b)

Fig. 8 Material with power law drag stress $\left(D(p)=\sigma_{Y}+k \sqrt{p}\right)$ and rate-dependency exponent $n=0.5$ under uniaxial tension $\left(\dot{\varepsilon}_{11}=1 \mathrm{~s}^{-1}\right.$ ). (a) Convergence plots for varying $\theta$. The convergence is linear for all values of $\theta$, except for $\theta=\theta^{*}$ at large time steps. (b) The final error on $p$ is minimized choosing $\theta \approx \theta^{*}$.

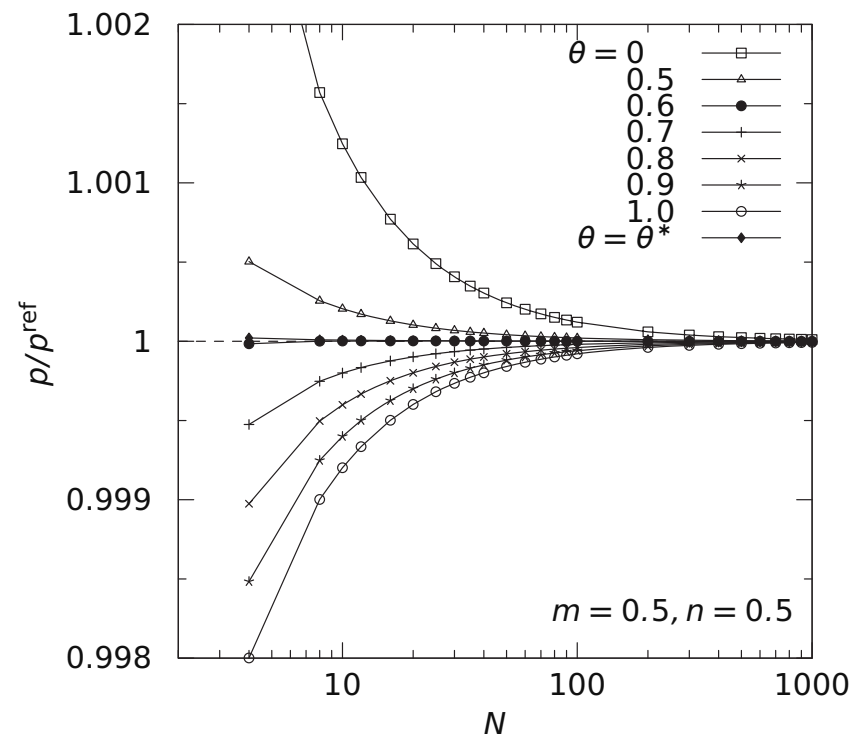

(a)

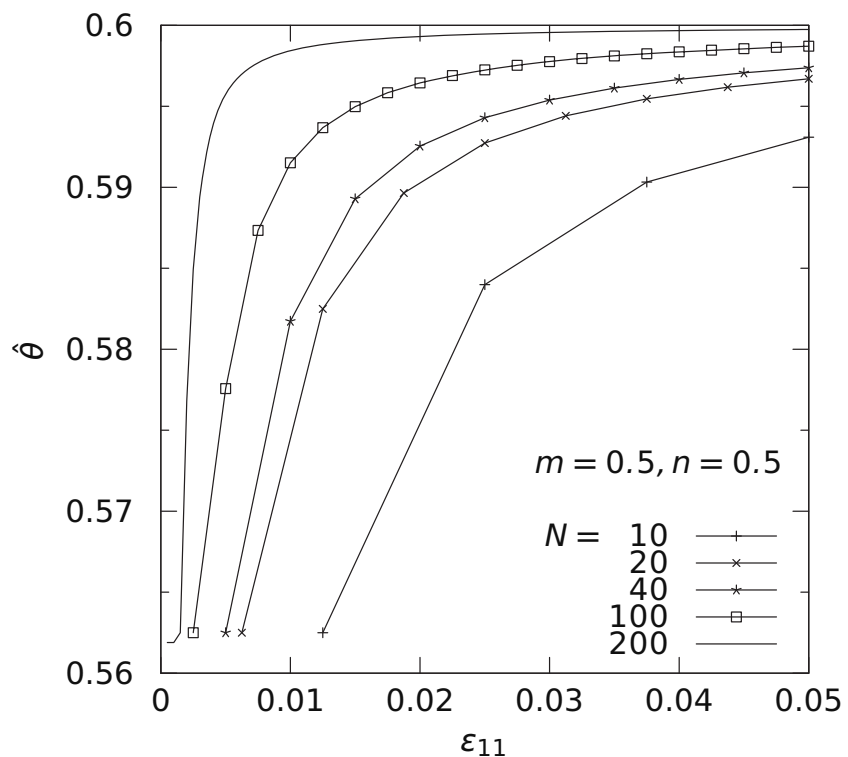

(b)

Fig. 9 Material with power law drag stress $(m=0.5)$ and rate-dependency exponent $n=0.5$ under uniaxial tension. (a) Evolution of the ratio $p / p^{\text {ref }}$ with $N$ for varing $\theta$. (b) Evolution of optimal integration parameter $\hat{\theta}$ with loading for varying number of load increments. 
combined with strain hardening, described by dissipation functions different from (15), a similar analysis could be carried out in order to measure the effect of the integration parameter. There may not always be a closed-form expression for the optimal value of this integration parameter, though.

Note that a large part of the analysis carried above stays valid within the framework of finite strains. In particular, as shown in [8], finite strain kinematic aspects can be decoupled from plastic strain update under the assumption of an isotropic elastic free energy quadratic in logarithmic (natural) strains. The radial return structure of variational updates and all the above discussion on the effect of the choice of $\theta$ then fully applies.

Acknowledgements LB is mandated by the Belgian Fund for Scientific Research (F.R.S.-FNRS).

\section{References}

[1] B. Halphen and Q.S. Nguyen. Sur les matériaux standard généralisés, J. Mécanique 14(1), 39-63 (1975).

[2] P. Germain, Q. S. Nguyen, and P. Suquet. Continuum thermodynamics, J. Appl. Mech. 50(4b), 1010-1020 (1983).

[3] P. Mialon. Eléments d'analyse et de résolution numérique des relations de l'élasto-plasticité, EDF Bulletin de la Direction des Etudes et Recherches Série C, 3, 57-89 (1986).

[4] C. Comi, A. Corigliano, and G. Maier. Extremum properties of finite-step solutions in elastoplasticity with nonlinear hardening, Int. J. Solids Struct. 27(8), 965-981 (1991).

[5] J. Martin, M. Kaunda, and R. Isted. Internal variable formulations of elastic-plastic dynamic problems, Int. J. Impact Engng. 18(7-8), 849-858 (1996).

[6] A. Carini. Colonnetti's minimum principle extension to generally non-linear materials, Int. J. Solids Struct. 33(1), 121-144 (1996).

[7] C. Miehe. Strain-driven homogenization of inelastic microstructures and composites based on an incremental variational formulation, Int. J. Numer. Meth. Engng. 55(11), 1285-1322 (2002).

[8] M. Ortiz and L. Stainier. The variational formulation of viscoplastic constitutive updates, Comput. Methods Appl. Mech. Engrg. 171(3-4), 419-444 (1999).

[9] J. Mosler and M. Ortiz. Variational h-adaption in finite deformation elasticity and plasticity, Int. J. Numer. Meth. Engng. 72(5), 505-523 (2007).

[10] J. Mosler and M. Ortiz. An error-estimate-free and remapping-free variational mesh refinement and coarsening method for dissipative solids at finite strains, Int. J. Numer. Meth. Engng. 77(3), 437-450 (2009).

[11] N. Lahellec and P. Suquet. On the effective behavior of nonlinear inelastic composites: I. Incremental variational principles, J. Mech. Phys. Solids 55(9), 1932-1963 (2007).

[12] N. Lahellec and P. Suquet. On the effective behavior of nonlinear inelastic composites: II. A second-order procedure, J. Mech. Phys. Solids 55(9), 1964-1992 (2007).

[13] L. Brassart. Homogenization of elasto-(visco)plastic composites: history-dependent incremental and variational approaches, $\mathrm{PhD}$ thesis, École polytechnique de Louvain, 2011.

[14] J. Mosler and O. Bruhns. Towards variational constitutive updates for non-associative plasticity models at finite strain: Models based on a volumetric-deviatoric split, Int. J. Solids Struct. 46(78), 1676 - 1684 (2009).

[15] J. Mosler. Variationally consistent modeling of finite strain plasticity theory with non-linear kinematic hardening, Comput. Methods Appl. Mech. Engrg. 199(45-48), 2753 - 2764 (2010).

[16] E. Fancello, J.P. Ponthot, and L. Stainier. A variational formulation of constitutive models and updates in non-linear finite viscoelasticity, Int. J. Numer. Meth. Engng. 65(11), 1831-1864 (2006). 
[17] Q. Yang, L. Stainier, and M. Ortiz. A variational formulation of the coupled thermo-mechanical boundary-value problem for general dissipative solids, J. Mech. Phys. Solids 54(2), 401-424 (2006).

[18] M. L. Wilkins. Calculation of elasto-plastic flow, in: Methods of computational physics (vol. 3), edited by B. Alder et al. (Academic Press, New York, 1964), pp. 211-263.

[19] L. Stainier. Consistent incremental approximation of dissipation pseudo-potentials in the variational formulation of thermo-mechanical constitutive updates, Mech. Res. Commun. 38(4), 315319 (2011).

[20] J. Lemaître and J. L. Chaboche. Mechanics of solid materials (Cambridge Univ. Press, 1990).

[21] G. A. Maugin. The thermomechanics of plasticity and fracture (Cambridge Univ. Press, 1992).

[22] L. Stainier and M. Ortiz. Study and validation of a variational theory of thermomechanical coupling in finite viscoplasticity, Int. J. Solids Struct. 47(5), 705-715 (2010).

[23] J. L. Chaboche. A review of some plasticity and viscoplasticity constitutive theories, Int. J. Plast. 24(10), 1642-1693 (2008).

[24] J. C. Simo and T. J. R. Hughes. Computational Inelasticity (Springer, 1998).

[25] I. Doghri. Mechanics of deformable solids: linear and nonlinear, analytical and computational aspects (Springer, 2000). 